\title{
The Ipsilesional Upper Limb Can Be Affected following Stroke
}

\author{
Gemma H. Kitsos, ${ }^{1}$ Isobel J. Hubbard, ${ }^{2}$ Alex R. Kitsos, ${ }^{3}$ and Mark W. Parsons ${ }^{1}$ \\ ${ }^{1}$ Stroke Research, Neurology Department, John Hunter Hospital, Hunter New England Local Health District, Locked Bag 1, \\ Hunter Regional Mail Centre, NSW 2310, Australia \\ ${ }^{2}$ School of Medicine and Public Health, University of Newcastle, Callaghan, NSW 2308, Australia \\ ${ }^{3}$ Hunter Brain Injury Service, Hunter New England Local Health District, Bar Beach, NSW 2300, Australia \\ Correspondence should be addressed to Gemma H. Kitsos; gemma.kitsos@hnehealth.nsw.gov.au
}

Received 20 August 2013; Accepted 17 September 2013

Academic Editors: A. Biegon and F. Gonzalez-Lima

Copyright ( $) 2013$ Gemma H. Kitsos et al. This is an open access article distributed under the Creative Commons Attribution License, which permits unrestricted use, distribution, and reproduction in any medium, provided the original work is properly cited.

\begin{abstract}
Objective. Neurological dysfunction commonly occurs in the upper limb contralateral to the hemisphere of the brain in which stroke occurs; however, the impact of stroke on function of the ipsilesional upper limb is not well understood. This study aims to systematically review the literature relating to the function of the ipsilesional upper limb following stroke and answer the following research question: Is the ipsilesional upper limb affected by stroke? Data Source. A systematic review was carried out in Medline, Embase, and PubMed. Review Methods. All studies investigating the ipsilesional upper limb following stroke were included and analysed for important characteristics. Outcomes were extracted and summarised. Results. This review captured 27 articles that met the inclusion criteria. All studies provided evidence that the ipsilesional upper limb can be affected following stroke. Conclusion. These findings demonstrate that clinicians should consider ipsilesional upper limb deficits in rehabilitation and address this reduced functional capacity. Furthermore, the ipsilesional upper limb should not be used as a "control" measure of recovery for the contralateral upper limb.
\end{abstract}

\section{Introduction}

Neurological dysfunction commonly occurs in the upper limb contralateral to the hemisphere of the brain in which stroke occurs; however, the effect on the ipsilesional upper limb (iUL) is poorly understood $[1,2]$. Contralateral deficits increase reliance on the iUL for function and for maintaining independence $[3,4]$. Recognising the impact of stroke on the iUL is an important step towards implementing effective rehabilitation and to improve our understanding of the challenges faced following stroke $[5,6]$.

Health professionals commonly use the iUL as a measure of reference for recover, and frequently refer to it as "nonaffected" or "unaffected" $[2,7]$. For health professionals to simply presume that the iUL is not affected by stroke, as our current terminology infers, may fail to adequately recognise the contribution of a functionally important component of upper limb recovery.

In this study, the term iUL refers to the arm and hand on the same side of the body as the lesioned hemisphere. This study will systematically review the research investigating the iUL following stroke to determine if the iUL is affected or not affected by stroke. This study hypothesises that following stroke the iUL can be adversely affected.

\section{Method}

2.1. Search Strategy. A systematic search of the literature was carried out in October 2012 using the following databases: Medline, Embase, and PubMed. Search strategies were developed in accordance with the requirement of each database to locate studies for inclusion. The following search terms were used: stroke, upper limb, upper extremity, arm, less affected, nonaffected, and ipsilateral. An example search strategy has been included (Table 2). A further manual search was conducted from the reference lists of the "captured" studies to identify other relevant studies for inclusion.

2.2. Inclusion/Exclusion Criteria. This review only included articles reporting original research that recruited adult stroke 


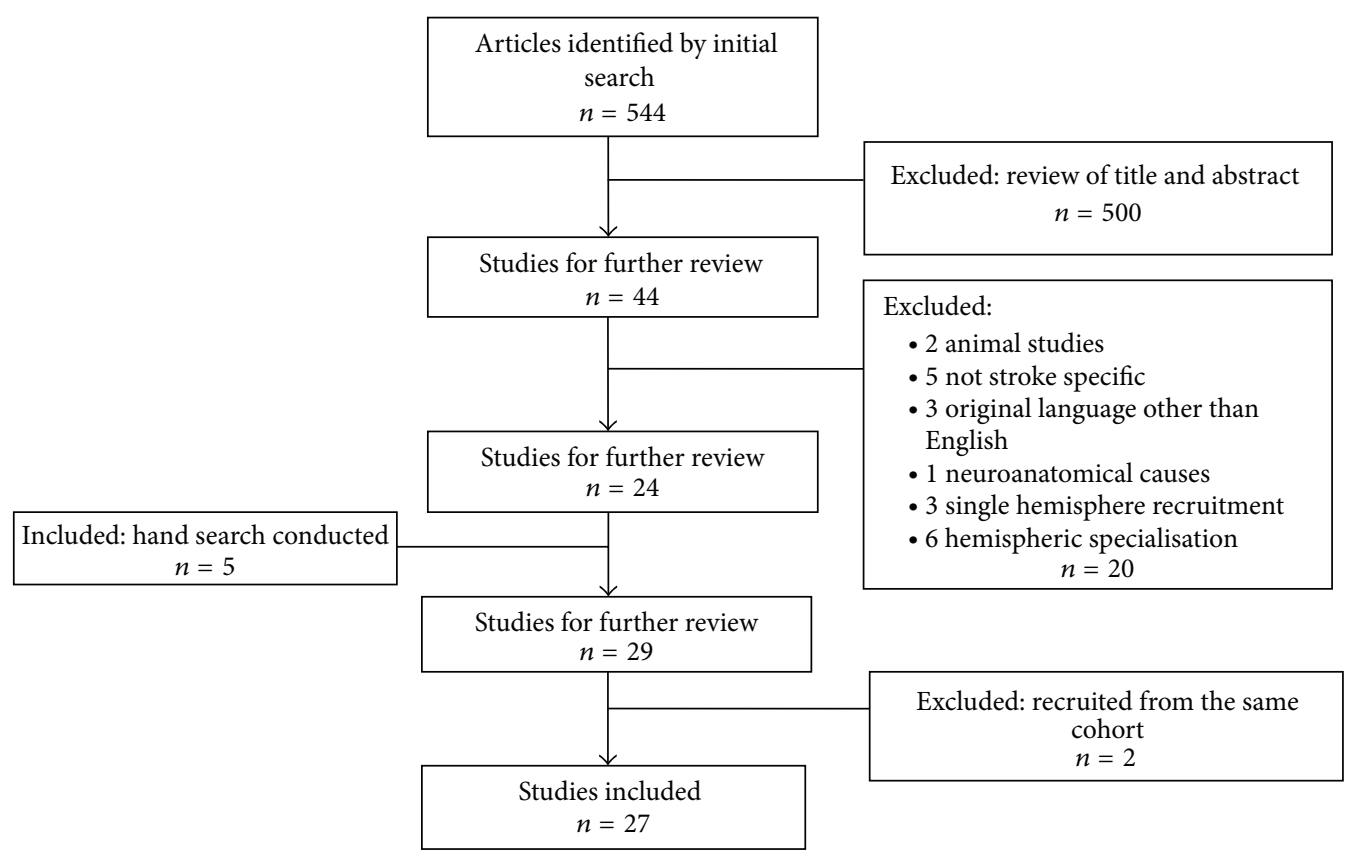

Figure 1: Selection of studies.

survivors. It excluded studies not initially published in English, conference publications, and those that used animal modelling. It also excluded studies which only recruited patients with a left or a right hemispheric stroke, studies which aimed to analyse the role of each hemisphere and/or the function of the iUL, and studies which explored neuroanatomical causes for iUL deficit (Figure 1).

2.3. Selection of Studies. From the initial search, titles and abstracts were reviewed for relevance. Studies which appeared to meet the inclusion criteria were then analysed using the full text. Once the inclusion criteria were confirmed, relevant data was then extracted by the review panel in accordance with a customised data collection form. Where discrepancies arose, the review panel reached agreement through discussion.

\section{Results}

This systematic review found that the iUL can be affected following stroke. The search captured 27 studies which assessed iUL motor and/or sensory deficits following stroke (Table 1).

The iUL was reported as affected in all of the 27 studies captured by this review. The publication dates ranged from 1971 to 2012 , with eight (29.6\%) studies published before the year 2000. The number of participants with stroke ranged from seven participants to 100; mean (SD) participant cohort was 33.2 (22.8) years. Participant ages ranged from 50.1 to 72.4 years; mean (SD) age was 60.7 (6.1). Isolated deficits of the iUL were not reported; contralateral upper limb deficits were present in all participants recruited to the stroke cohort across the 27 included studies.
Only Noskin et al. [1] and Spaulding et al. [8] compared a stroke cohort to normative data, whilst all the remaining studies $(n=25,92.6 \%)$ compared results to age-matched healthy controls. A mixed cohort of left-handed and righthanded participants was recruited in six studies, whilst 18 studies $(66.7 \%)$ recruited right-handed participants only. Hand dominance data was incomplete in the remaining three studies $[2,9,10]$.

Standardised assessments were utilised in 12 (44.5\%) studies to explore iUL deficits [1-8, 11-14]. Noskin et al. [1], Yelnik et al. [15], and Morris and Van Wijck [12] assessed upper limb function using the Nine Hole Peg Test (9HPT) [16], and Sunderland et al. [7], Wetter et al. [3], Jebsen et al. [14], and Spaulding et al. [8] utilised the Jebsen Hand Function Test (JHFT) [9]. Laufer et al. [4] assessed with both the 9HPT and the JHFT. The Action Research Arm Test was used by Morris and Van Wijck [12] and Nowak et al. [17].

A dynamometer was the most frequently used assessment tool to determine strength $(n=5,18.5 \%)[1,7$, $10,13,18$ ]. Noskin et al. [1] reported that grip strength was not significantly affected at the time points assessed: 24-48 hours, one week, three months, and one year after stroke. Sunderland et al. [7] reported that grip strength was reduced within one month of stroke $(P<0.001)$, and in a subsequent study [19] they reported that grip strength had significantly improved at six months after stroke. McCrea et al. [10] reported that 12 months after a stroke event, strength remained affected in the iUL $(P<0.001)$.

Both standardised and nonstandardised assessments were used in seven studies (25.9\%) [10, 15, 17, 18, 20-22]. A further eight studies (29.6\%) used only nonstandardised assessments and employed a case-control study design [2330]. When considering the primary outcome of the studies, Brasil-Neto and De Lima [13] focused on sensory deficits, 


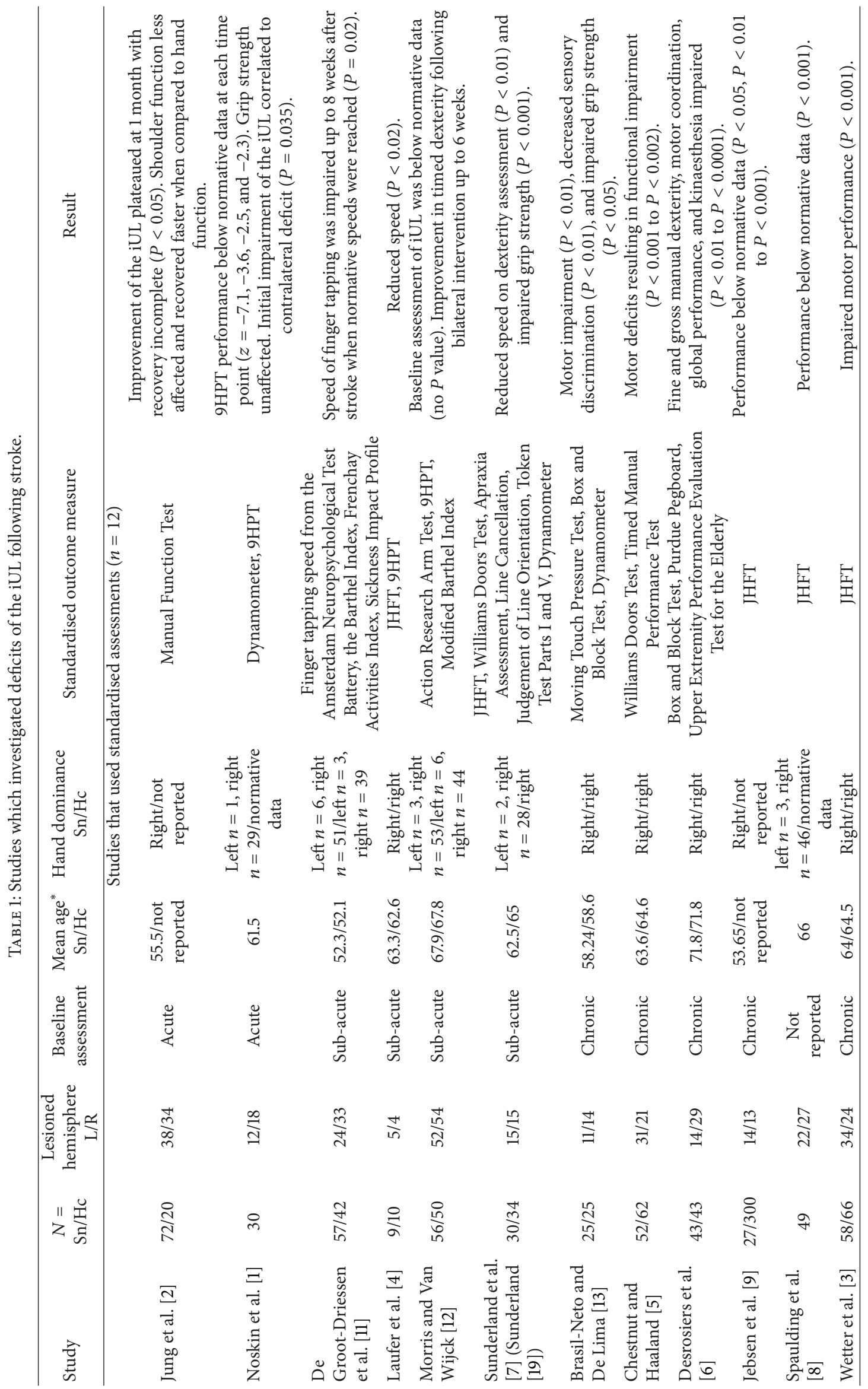




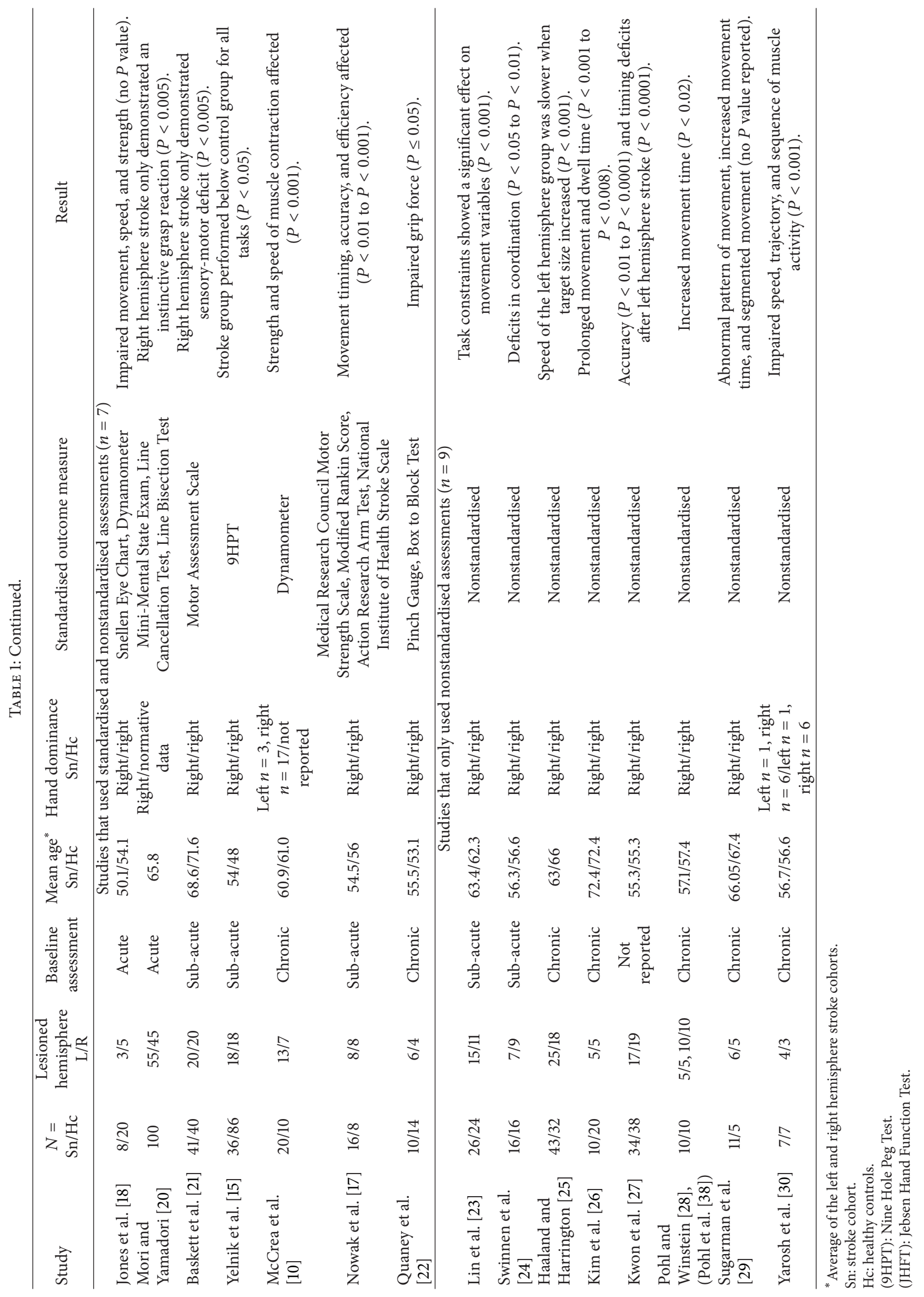


TABLE 2: Example search methodology: Embase from 1974 till present.

\begin{tabular}{lc}
\hline Search terms & Number of articles \\
\hline Stroke and upper limb; arm & 2167 \\
Less affected and stroke and upper limb; arm & 29 \\
Nonaffected and stroke and upper limb; arm & 14 \\
Ipsilateral and stroke and upper limb; arm & 85 \\
\hline
\end{tabular}

Sunderland et al. [7] investigated cognitive deficits, and the remaining studies measured motor deficits $(n=25,92.6 \%)$.

When considering time after stroke, four (14.8\%) studies $[1,2,18,20]$ recruited participants in the acute phase after stroke ( $\leq$ one week), nine (33.4\%) studies $[4,7,11,12,15,17$, $21,23,24]$ recruited participants in the subacute phase after stroke, and $12(44.5 \%)$ studies investigated a chronic stroke cohort ( $\geq$ six months). Baseline assessment was unable to be determined in further two studies $(7.4 \%)[8,27]$. Baskett et al. [21], Jung et al. [2], Noskin et al. [1], Laufer et al. [4], and De Groot-Driessen et al. [11] assessed change over multiple time points.

\section{Discussion}

This review found evidence that stroke can adversely affect the iUL. To our knowledge, this is the first systematic review of iUL performance to date. This review demonstrates that iUL deficits can be present in the acute, subacute, and chronic phases of stroke recovery. Of the 27 studies reviewed, eight were published before the year 2000 demonstrating that this is not a new concept in stroke research; however, despite current supportive evidence, it continues to be poorly recognised and understood [2,7]. This evidence challenges the current clinical vocabulary which refers to the "nonaffected" or "unaffected" iUL $[2,7]$. It also reinforces the fact that health professionals should not be using the iUL as a "control" measure for dysfunction in the contralateral upper limb.

This review has demonstrated that, as with the contralesional upper limb, there is a broad range of measures that can be used to assess iUL impairment. It provides evidence that the 9HPT $[1,4,12,15]$ and JHFT $[3,4,7-9,14]$ are sensitive to motor impairment in the iUL. Both assessments are used routinely in patients recovering from stroke, and the 9HPT has been validated for use in this cohort in a systematic literature review [31]. In contrast, grip strength of the iUL was reported to be both affected $[7,10]$ and unaffected $[1,18]$ across the acute, subacute, and chronic phases of stroke recovery. These conflicting results may be reflective of between-study differences in the participant cohort, or they may reflect the fact that this type of measure is, in fact, a crude de facto measure of corticospinal tract integrity [32].

4.1. Clinical Relevance. The current systematic review finds evidence of abnormal patterns of movement and strength in the iUL. These deficits can be linked to reduced functional capacity following stroke and may impact patient outcomes $[1,5,11]$. These findings indicate that clinicians need to assess, consider, and if relevant, treat impairment of the iUL to effectively manage upper limb rehabilitation after stroke. While the contralateral limb should remain the primary focus of upper limb rehabilitation, this review provides evidence to support the importance of bilateral interventions thereby addressing deficits of both the contralateral upper limb and the iUL [32].

The results of this review validate a change in upper limb vocabulary, and we recommend that the term "less affected" be used when referring to the iUL and the term "more affected" be used when referring to the contralateral upper limb. Health professionals should cease using the terms "unaffected" and "nonaffected" to describe the iUL following stroke, as these terms are misleading at best.

4.2. Study Limitations. Heterogeneity between studies is a limitation of this systematic review. Table 1 illustrates the variances of cohort characteristics. Lesion size and location are not documented in this review; however, it is worth noting that while some researchers took this into consideration when recruiting participants, some made no mention of this at all. The time from stroke onset was another notable limitation with the majority of participants recruited in the chronic phase of stroke ( $\geq$ six months). Handedness was another between-study inconsistency. Right handed cohorts made up the majority of participants across studies with only some studies considering the association between upper limb function and handedness by comparing the iUL to the equivalent upper limb in control groups.

4.3. Further Research. Sunderland et al. [7] are the only authors who describe the effect of cognition on the deficits of the iUL. Therefore, further research is required to establish the impact of cognition on performance of the iUL. Further research is also needed to explore the pathophysiological mechanisms underpinning deficits of the iUL following stroke, and the role each hemisphere may play in the particular deficits exhibited.

4.4. Pathophysiological Mechanisms. The pathophysiological mechanisms which result in deficits of the iUL are largely unknown. Evidence at this time suggests various hypotheses; however, further research is needed to provide a definitive explanation. A dominant theory suggests that the ipsilesional uncrossed descending corticospinal pathways may play a role in the movement of the iUL [33]. Alternatively, a body of evidence supports the importance of interhemispheric, transcallosal interactions [17,34-37]. This suggests that activation of the ipsilateral hemisphere during unilateral upper limb movements might be related to excitatory or inhibitory effects in the contralateral hemisphere [35-38].

\section{Conclusion}

This systematic review finds that people who have experienced stroke can have a deficit of the iUL. Therefore, function in the iUL must be considered in rehabilitation to ensure maximum recovery and opportunities for increased 
independence. The clinical community needs to update the terminology associated with the iUL to acknowledge that it can be adversely affected and that it should not be used as a benchmark for recovery of function in the contralateral upper limb. While the contralateral limb should remain the primary focus of upper limb rehabilitation, this review provides evidence to support the importance of bilateral interventions thereby addressing deficits of both the contralateral upper limb and the iUL.

\section{References}

[1] O. Noskin, J. W. Krakauer, R. M. Lazar et al., "Ipsilateral motor dysfunction from unilateral stroke: implications for the functional neuroanatomy of hemiparesis," Journal of Neurology, Neurosurgery and Psychiatry, vol. 79, no. 4, pp. 401-406, 2008.

[2] H. Y. Jung, J. S. Yoon, and B. S. Park, "Recovery of proximal and distal arm weakness in the ipsilateral upper limb after stroke," NeuroRehabilitation, vol. 17, no. 2, pp. 153-159, 2002.

[3] S. Wetter, J. L. Poole, and K. Y. Haaland, "Functional implications of ipsilesional motor deficits after unilateral stroke," Archives of Physical Medicine and Rehabilitation, vol. 86, no. 4, pp. 776-781, 2005.

[4] Y. Laufer, L. Gattenio, E. Parnas, D. Sinai, Y. Sorek, and R. Dickstein, "Time-related changes in motor performance of the upper extremity ipsilateral to the side of the lesion in stroke survivors," Neurorehabilitation and Neural Repair, vol. 15, no. 3, pp. 167-172, 2001.

[5] C. Chestnut and K. Y. Haaland, "Functional significance of ipsilesional motor deficits after unilateral stroke," Archives of Physical Medicine and Rehabilitation, vol. 89, no. 1, pp. 62-68, 2008.

[6] J. Desrosiers, D. Bourbonnais, G. Bravo, P.-M. Roy, and M. Guay, "Performance of the "unaffected" upper extremity of elderly stroke patients," Stroke, vol. 27, no. 9, pp. 1564-1570, 1996.

[7] A. Sunderland, M. P. Bowers, S.-M. Sluman, D. J. Wilcock, and M. E. Ardron, "Impaired dexterity of the ipsilateral hand after stroke and the relationship to cognitive deficit," Stroke, vol. 30, no. 5, pp. 949-955, 1999.

[8] S. J. Spaulding, J. J. McPherson, E. Strachota, M. Kuphal, and M. Ramponi, "Jebsen Hand Function Test: performance of the uninvolved hand in hemiplegia and of right-handed, right and left hemiplegic persons," Archives of Physical Medicine and Rehabilitation, vol. 69, no. 6, pp. 419-422, 1988.

[9] R. H. Jebsen, N. Taylor, R. B. Trieschmann, M. J. Trotter, and L. A. Howard, "An objective and standardized test of hand function," Archives of Physical Medicine and Rehabilitation, vol. 50, no. 6, pp. 311-319, 1969.

[10] P. H. McCrea, J. J. Eng, and A. J. Hodgson, "Time and magnitude of torque generation is impaired in both arms following stroke," Muscle and Nerve, vol. 28, no. 1, pp. 46-53, 2003.

[11] D. De Groot-Driessen, P. Van De Sande, and C. Van Heugten, "Speed of finger tapping as a predictor of functional outcome after unilateral stroke," Archives of Physical Medicine and Rehabilitation, vol. 87, no. 1, pp. 40-44, 2006.

[12] J. H. Morris and F. Van Wijck, "Responses of the less affected arm to bilateral upper limb task training in early rehabilitation after stroke: a randomized controlled trial," Archives of Physical Medicine and Rehabilitation, vol. 93, pp. 1129-1137, 2012.
[13] J. P. Brasil-Neto and A. C. De Lima, "Sensory deficits in the unaffected hand of hemiparetic stroke patients," Cognitive and Behavioral Neurology, vol. 21, no. 4, pp. 202-205, 2008.

[14] R. H. Jebsen, E. R. Griffith, E. W. Long, and R. Fowler, "Function of "normal" hand in stroke patients," Archives of Physical Medicine and Rehabilitation, vol. 52, no. 4, pp. 170-174, 1971.

[15] A. Yelnik, I. Bonan, M. Debray, E. Lo, F. Gelbert, and B. Bussel, "Changes in the execution of a complex manual task after ipsilateral ischemic cerebral hemispheric stroke," Archives of Physical Medicine and Rehabilitation, vol. 77, no. 8, pp. 806-810, 1996.

[16] K. O. Grice, K. A. Vogel, V. Le, A. Mitchell, S. Muniz, and M. A. Vollmer, "Adult norms for a commercially available nine hole peg test for finger dexterity," American Journal of Occupational Therapy, vol. 57, no. 5, pp. 570-573, 2003.

[17] D. A. Nowak, C. Grefkes, M. Dafotakis, J. Küst, H. Karbe, and G. R. Fink, "Dexterity is impaired at both hands following unilateral subcortical middle cerebral artery stroke," European Journal of Neuroscience, vol. 25, no. 10, pp. 3173-3184, 2007.

[18] R. D. Jones, I. M. Donaldson, and P. J. Parkin, "Impairment and recovery of ipsilateral sensory-motor function following unilateral cerebral infarction," Brain, vol. 112, no. 1, pp. 113-132, 1989.

[19] A. Sunderland, "Recovery of ipsilateral dexterity after stroke," Stroke, vol. 31, no. 2, pp. 430-433, 2000.

[20] E. Mori and A. Yamadori, "Unilateral hemispheric injury and ipsilateral instinctive grasp reaction," Archives of Neurology, vol. 42, no. 5, pp. 485-488, 1985.

[21] J. J. Baskett, H. J. Marshall, J. B. Broad, P. H. Owen, and G. Green, "The good side after stroke: ipsilateral sensory-motor function needs careful assessment," Age and Ageing, vol. 25, no. 3, pp. 239-244, 1996.

[22] B. M. Quaney, S. Perera, R. Maletsky, C. W. Luchies, and R. J. Nudo, "Impaired grip force modulation in the ipsilesional hand after unilateral middle cerebral artery stroke," Neurorehabilitation and Neural Repair, vol. 19, no. 4, pp. 338-349, 2005.

[23] K.-C. Lin, C.-Y. Wu, K.-H. Lin, and C.-W. Chang, "Effects of task instructions and target location on reaching kinematics in people with and without cerebrovascular accident: a study of the less-affected limb," American Journal of Occupational Therapy, vol. 62, no. 4, pp. 456-465, 2008.

[24] S. P. Swinnen, F. Debaere, D. Van Assche, C. Kiekens, and S. M. P. Verschueren, "Coordination of upper and lower limb segments: deficits on the ipsilesional side after unilateral stroke," Experimental Brain Research, vol. 141, no. 4, pp. 519-529, 2001.

[25] K. Y. Haaland and D. L. Harrington, "Limb-sequencing deficits after left but not right hemisphere damage," Brain and Cognition, vol. 24, no. 1, pp. 104-122, 1994.

[26] S. H. Kim, P. S. Pohl, C. W. Luchies, A. P. Stylianou, and Y. Won, "Ipsilateral deficits of targeted movements after stroke," Archives of Physical Medicine and Rehabilitation, vol. 84, no. 5, pp. 719724, 2003.

[27] Y.-H. Kwon, C. S. Kim, and S. H. Jang, "Ipsi-lesional motor deficits in hemiparetic patients with stroke," NeuroRehabilitation, vol. 22, no. 4, pp. 279-286, 2007.

[28] P. S. Pohl and C. J. Winstein, "Practice effects on the less-affected upper extremity after stroke," Archives of Physical Medicine and Rehabilitation, vol. 80, no. 6, pp. 668-675, 1999.

[29] H. Sugarman, A. Avni, R. Nathan, A. Weisel-Eichler, and J. Tiran, "Movement in the ipsilesional hand is segmented following unilateral brain damage," Brain and Cognition, vol. 48, no. 2-3, pp. 579-587, 2002. 
[30] C. A. Yarosh, D. S. Hoffman, and P. L. Strick, "Deficits in movements of the wrist ipsilateral to a stroke in hemiparetic subjects," Journal of Neurophysiology, vol. 92, no. 6, pp. 3276$3285,2004$.

[31] G. Kitsos, D. Harris, M. Pollack, and I. J. Hubbard, “Assessments in Australian stroke rehabilitation units: a systematic review of the post-stroke validity of the most frequently used," Disability and Rehabilitation, vol. 33, no. 25-26, pp. 2620-2632, 2011.

[32] J. W. Stinear and W. D. Byblow, "Rhythmic bilateral movement training modulates corticomotor excitability and enhances upper limb motricity poststroke: a pilot study," Journal of Clinical Neurophysiology, vol. 21, no. 2, pp. 124-131, 2004.

[33] U. Ziemann, K. Ishii, A. Borgheresi et al., "Dissociation of the pathways mediating ipsilateral and contralateral motorevoked potentials in human hand and arm muscles," Journal of Physiology, vol. 518, no. 3, pp. 895-906, 1999.

[34] C. Grefkes and G. R. Fink, "Reorganization of cerebral networks after stroke: new insights from neuroimaging with connectivity approaches," Brain, vol. 134, no. 5, pp. 1264-1276, 2011.

[35] T. Shimizu, A. Hosaki, T. Hino et al., "Motor cortical disinhibition in the unaffected hemisphere after unilateral cortical stroke," Brain, vol. 125, no. 8, pp. 1896-1907, 2002.

[36] N. S. Ward and L. G. Cohen, "Mechanisms underlying recovery of motor function after stroke," Archives of Neurology, vol. 61, no. 12, pp. 1844-1848, 2004.

[37] C. M. Stinear, P. A. Barber, P. R. Smale, J. P. Coxon, M. K. Fleming, and W. D. Byblow, "Functional potential in chronic stroke patients depends on corticospinal tract integrity," Brain, vol. 130, no. 1, pp. 170-180, 2007.

[38] P. S. Pohl, C. W. Luchies, J. Stoker-Yates, and P. W. Duncan, "Upper extremity control in adults post stroke with mild residual impairment," Neurorehabilitation and Neural Repair, vol. 14, no. 1, pp. 33-41, 2000. 


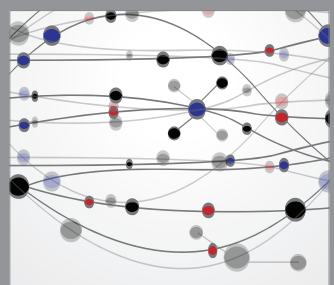

The Scientific World Journal
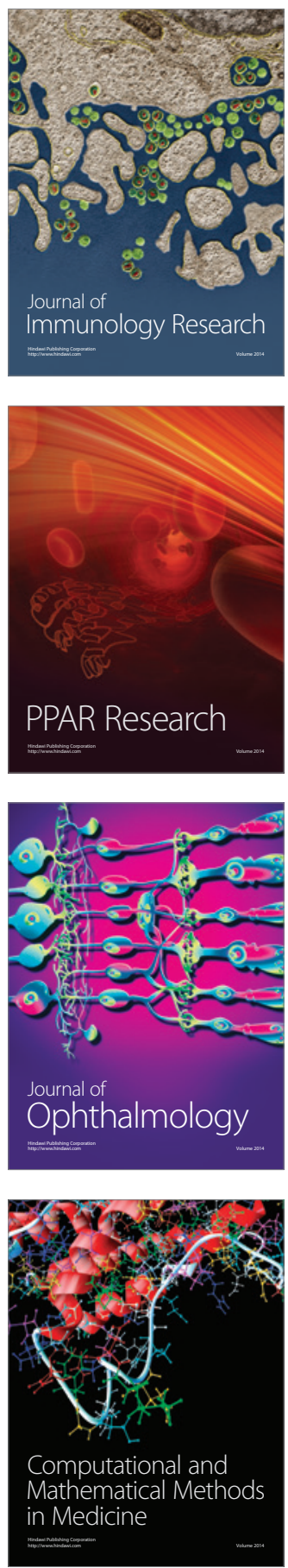

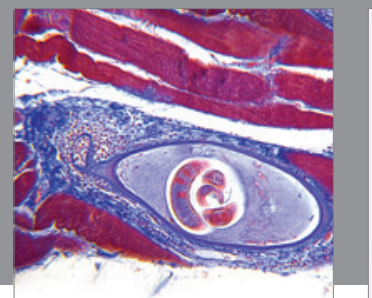

Gastroenterology

Research and Practice
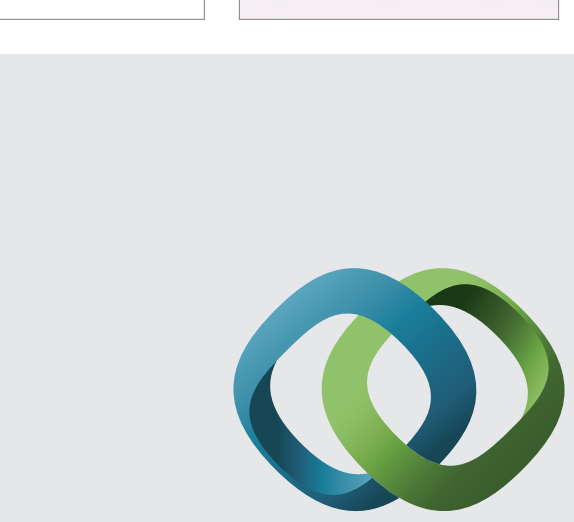

\section{Hindawi}

Submit your manuscripts at

http://www.hindawi.com
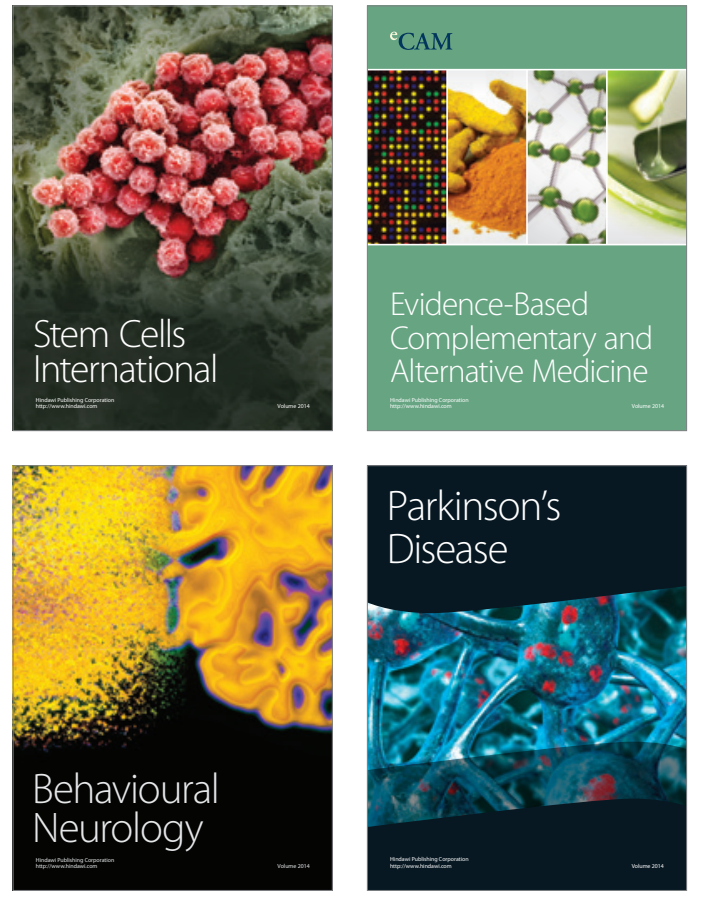
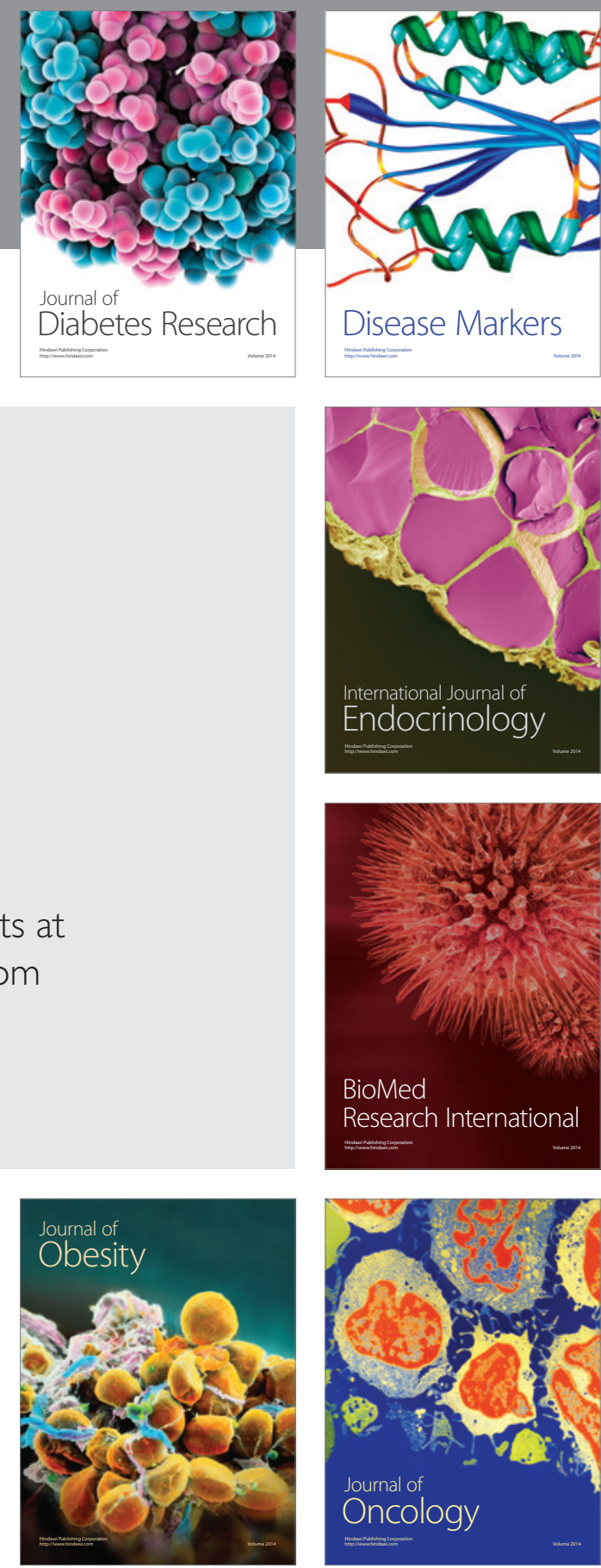

Disease Markers
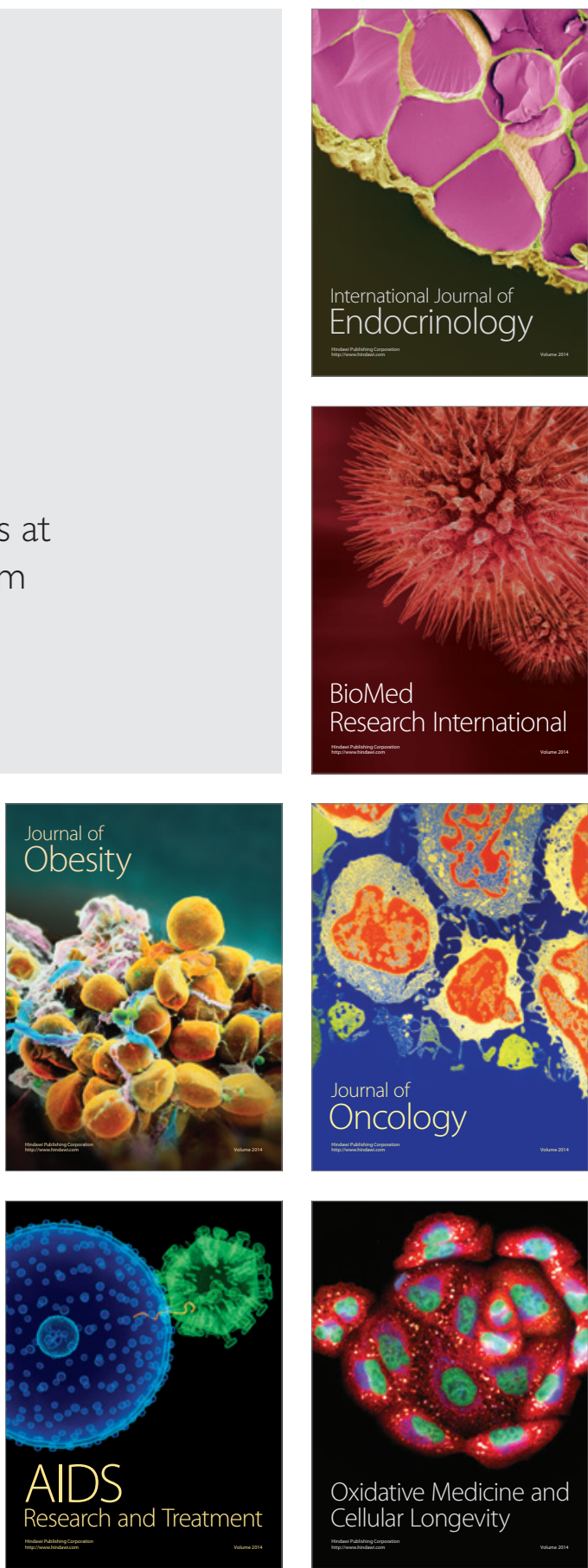